\title{
Eventración diafragmática congénita en un paciente de nueve meses: presentación de caso clínico y revisión de la literatura
}

\author{
Carlos Roberto Guzmán-Valderrábano,凶 Raúl Hernández-Saldaña, Mario Soto-Ramos, \\ Luis Carlos Hinojos-Gallardo
}

Hospital Infantil de Especialidades del Estado de Chihuahua, Chihuahua, México.

Trabajo recibido: 29-III-2016; aceptado: 20-X-2016

RESUMEN. La eventración diafragmática es una patología infrecuente en la edad pediátrica, definida como una elevación anormal, ya sea parcial o total del diafragma, sin una solución de la continuidad asociada con el desplazamiento de los órganos abdominales hacia el tórax. Algunos reportes refieren una incidencia de 1-3/1,000 recién nacidos. Se presenta con mayor frecuencia en el sexo masculino y representa el $5 \%$ de todas las anomalías diafragmáticas. Según su origen se clasifican en congénita y adquirida teniendo cada una de ellas características particulares. Puede presentarse de manera aislada o asociada con otros defectos congénitos como: hipoplasia pulmonar, enfermedad cardíaca congénita, pectum excavatum, paladar hendido, hipospadias, criptorquidia y tortícolis congénita. El diagnóstico se realiza con una radiografía de tórax posteroanterior y una lateral en donde se muestre una elevación del hemidiafragma derecho al menos dos espacios intercostales por arriba del izquierdo, o el izquierdo elevado un espacio intercostal por arriba del derecho. El tratamiento de la ED difiere dependiendo de su origen y manifestaciones clínicas, siendo en algunos casos necesario el manejo quirúrgico por lo que se presenta un caso clínico así como la revisión de la literatura.

Palabras clave: Eventración diafragmática, eventración diafragmática congénita, plicatura diafragmática, toracotomía posterolateral.

ABSTRACT. Eventration of the diaphragm is a rare disease in children, defined as an abnormal elevation, either partially or totally, of the diaphragm without a solution of continuity associated with the displacement of the abdominal organs into the chest. Some reports refer an incidence of 1 to $3 / 1,000$ newborns. It occurs more frequently in males, and represents $5 \%$ of all diaphragmatic abnormalities. They are classified according to their origin in congenital and acquired each having particular characteristics. It can occur in isolation or associated with other birth defects: pulmonary hypoplasia, congenital heart disease, pectus excavatum, cleft palate, hypospadias, cryptorchidism and congenital torticollis. The diagnosis is made with a chest film and a side where an elevated right hemidiaphragm is displayed at least two intercostal spaces above the left, or left intercostal space high above the law. The treatment of ED differs depending on its origin and clinical manifestations being in some cases required surgical management so a case report and review of the literature is presented.

Key words: Eventration of the diaphragm, congenital eventration of the diaphragm, diaphragmatic plication, posterolateral thoracotomy.

\section{INTRODUCCIÓN}

La eventración diafragmática (ED) es una patología de presentación poco frecuente en la edad pediátrica, se define como una elevación anormal ya sea parcial o total del diafragma sin solución de la continuidad del mismo y con desplazamiento de las vísceras de la cavidad abdominal hacia el tórax.1,2

Una gran parte de estos pacientes cursarán asintomáticos incluso hasta la edad adulta y suelen ser diagnosticados al realizarse algún estudio radiológico del tórax por otra circunstancia. En cuanto a su incidencia, la literatura refiere que se presenta de 1-3/1,000 recién nacidos vivos, siendo más frecuente en el sexo masculino y representa el $5 \%$ de las patologías del diafragma. ${ }^{3,4}$ Por su etiología es posible clasificarlas en congénita o adquirida, siendo la primera una causa aún menos frecuente, por lo que presentamos el caso clínico de una paciente con diagnóstico de ED congénita, la cual fue diagnosticada posterior a presentar cuadro agudo de dificultad respiratoria sin causa aparente.

\section{PRESENTACIÓN DEL CASO}

Paciente masculino de 9 meses de edad, originario y residente de Chihuahua, Chihuahua. Hijo de madre de 
30 años al momento del embarazo, inició control prenatal desde el primer trimestre de embarazo, cursó con embarazo de evolución normal, se realizaron dos ultrasonidos reportados normales, a las 39 semanas de gestación nace vía cesárea por cesárea iterativa, llora y respira al nacer, se realizaron maniobras básicas de reanimación neonatal, con peso al nacimiento de $3600 \mathrm{gr}$, talla: 50 $\mathrm{cm}$, Apgar desconocido. Egresado con la madre a las 48 horas de vida sin complicaciones, sin otros antecedentes personales o familiares de importancia.

Inició su padecimiento aproximadamente cuatro horas previas a su ingreso presentando de manera súbita tos seca, posteriormente flemosa, dificultad para respirar, vómito y cianosis; 15 minutos más tarde

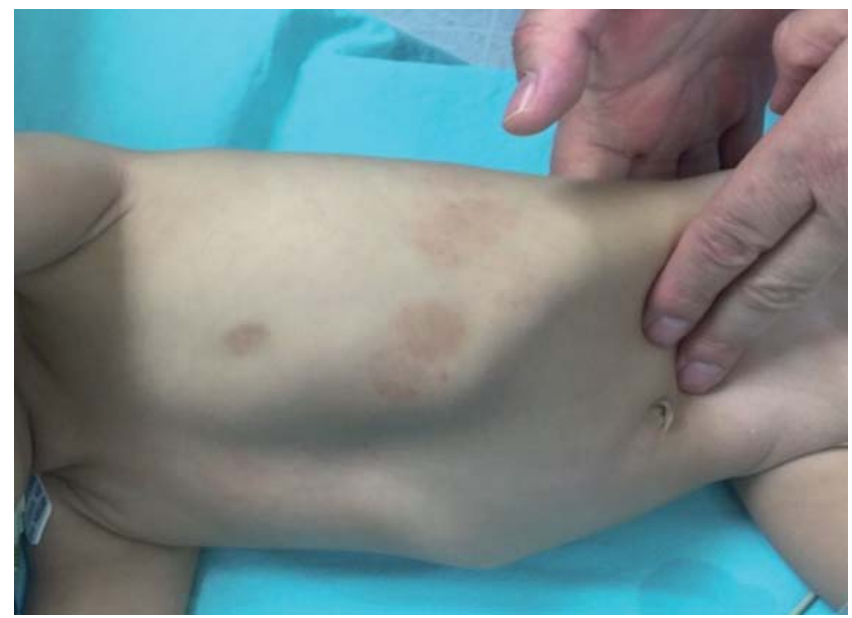

Figura 1. Exploración física se observa abdomen excavado o en batea. presenta nuevamente misma sintomatología por lo que acude al Servicio de Urgencias. Durante su valoración en urgencias se encontró en la exploración física tórax normolineo con movimientos de amplexión y amplexación normales, auscultación pulmonar con presencia de ruidos peristálticos en región basal de hemitórax izquierdo, resto de campos pulmonares con ruidos respiratorios normales, el abdomen estaba en batea (figura 1), blando, depresible, con ruidos peristálticos disminuidos en intensidad y frecuencia, sin datos de irritación peritoneal ni visceromegalias. Se realizó radiografía toracoabdominal anteroposterior y lateral de tórax (figura 2) donde se encuentran múltiples imágenes radiolúcidas de bordes bien delimitados, así como elevación del hemidiafragma izquierdo hasta quinto espacio intercostal izquierdo, desplazamiento del mediastino hacia el hemitórax derecho y ausencia de cámara gástrica y asas intestinales en abdomen.

Es valorado por el Servicio de Neumología Pediátrica y Cirugía de Tórax estableciendo el diagnóstico de ED, razón por la cual se decide su hospitalización para manejo quirúrgico. Se realiza toracotomía lateral izquierda a nivel del séptimo espacio intercostal encontrando protrusión del hemidiafragma izquierdo, se lleva a cabo corte e invaginación del mismo y se coloca una malla sintética (forma oval) de $4 \mathrm{~cm}$ de diámetro corrigiendo el defecto diafragmático (figura 3); durante la cirugía se realiza maniobra de inspiración observándose el pulmón macroscópicamente de tamaño y características normales. Se establece diagnóstico posoperatorio de ED congénita sin hipoplasia pulmonar.

En el período posquirúrgico ingresó a la sala de Cuidados Intermedios donde permaneció 48 horas
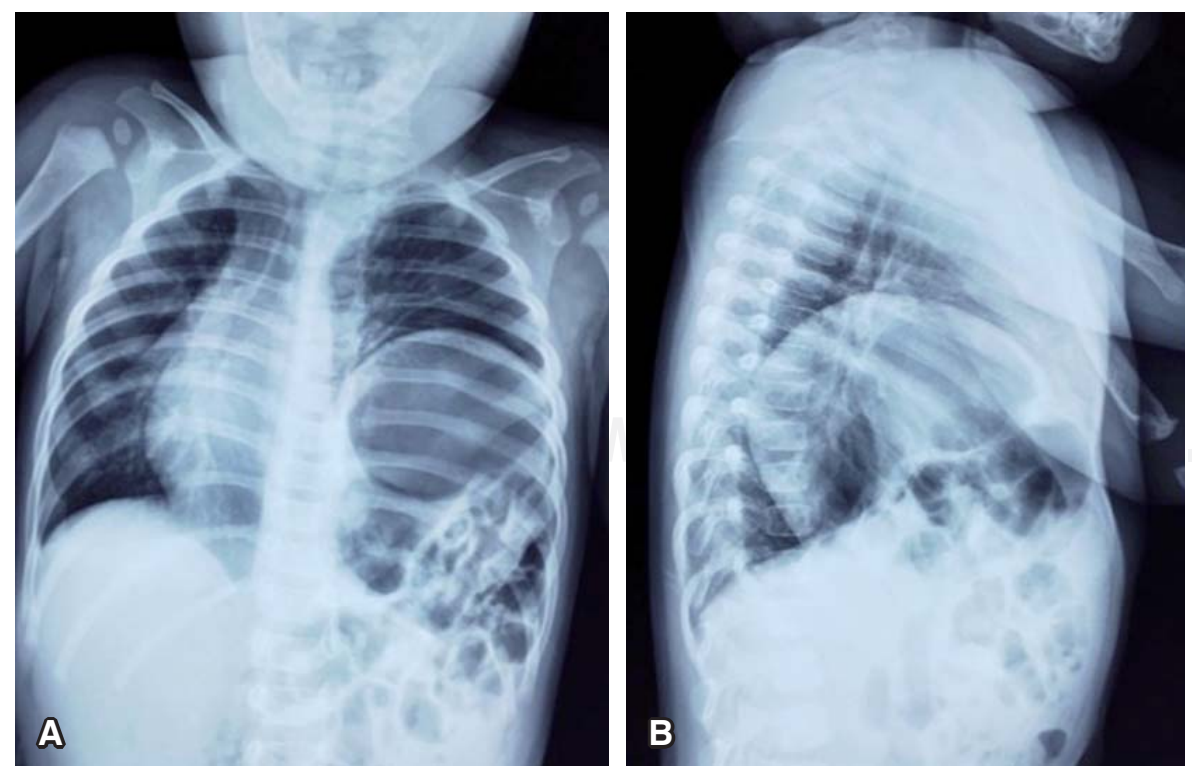

Figura 2.

Radiografías de tórax al ingreso. Panel A: proyección anteroposterior. Panel B: proyección lateral izquierda. Se observa elevación del hemidiafragma izquierdo hasta quinto espacio intercostal y desplazamiento del área mediastinal hacia hemitórax derecho. 

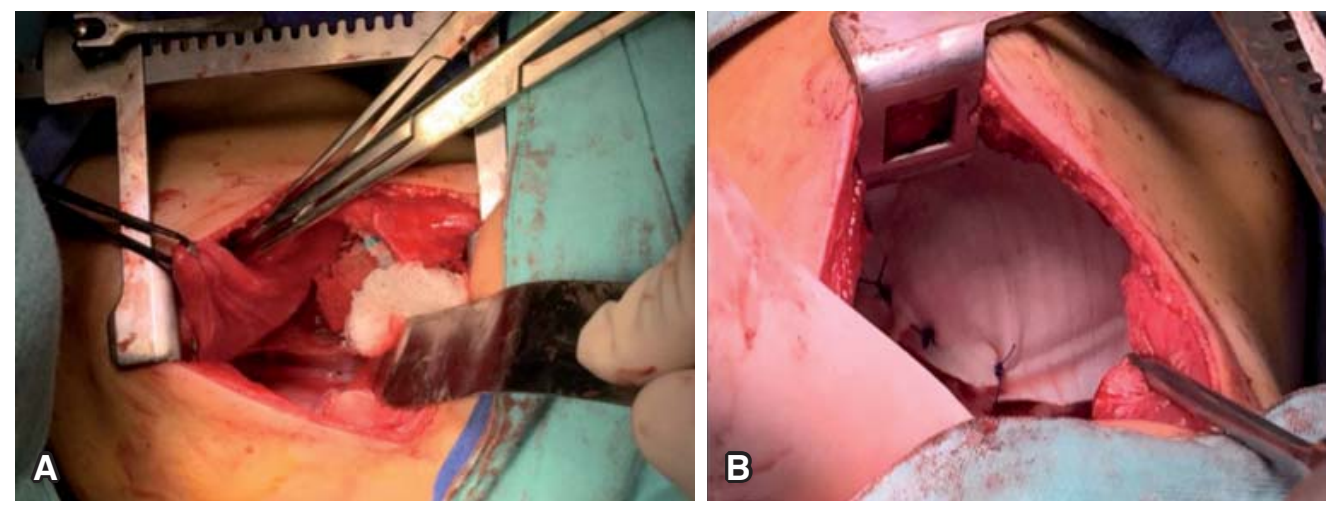

Figura 3.

Toracotomía lateral izquierda. Panel A: diafragma izquierdo flácido. Panel B: invaginación y colocación de malla sintética en diafragma izquierdo.

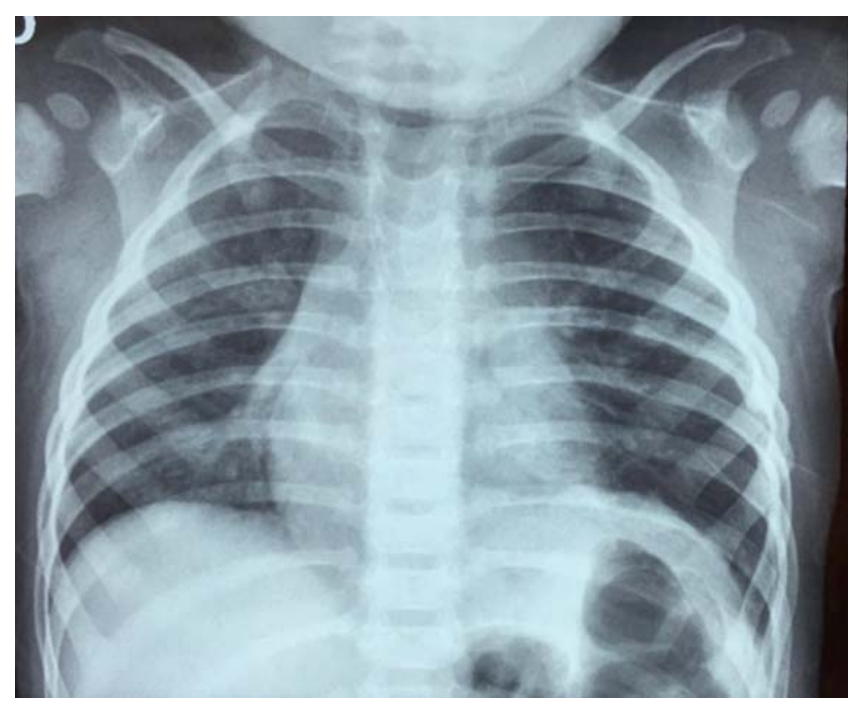

Figura 4. Radiografía de tórax simple a las 72 horas del posquirúrgico.

cursando con evolución favorable; posterior a ello, pasó a la sala General de Pediatría, se le retira la sonda pleural a las 72 horas posteriores al evento quirúrgico, se tomó radiografía posteroanterior de tórax en la cual pudo apreciarse hemidiafragma izquierdo en posición normal y adecuada expansión de parénquima pulmonar (figura 4). En su séptimo día de estancia hospitalaria fue egresado continuando su seguimiento por Consulta Externa de Neumología Pediátrica y Cirugía de Tórax.

\section{DISCUSIÓN}

La eventración diafragmática (ED) es una patología infrecuente en la edad pediátrica. Es definida como una elevación anormal, ya sea parcial o total del diafragma sin una solución de la continuidad asociada con el desplazamiento de las vísceras de la cavidad abdominal hacia el tórax. ${ }^{1}$ En la mayor parte de los casos, los pacientes con este tipo de patologías cursan asintomáticos incluso hasta la edad adulta y casi siempre son diagnosticados al realizarse una radiografía de tórax por algún otro motivo. ${ }^{2}$ En el paciente presentado el hallazgo de la patología fue incidental.

Su incidencia real es desconocida; aunque se considera como una patología poco frecuente en pediatría. Existen algunos reportes que muestran una incidencia de 1-3/1,000 recién nacidos vivos. ${ }^{3}$ Es más frecuente en el sexo masculino y representa el $5 \%$ de todas las anomalías diafragmáticas. ${ }^{4}$ En las eventraciones izquierdas, el estómago puede invertirse bajo la cúpula diafragmática con el riesgo de alteraciones del vaciamiento gástrico, de disfagia o de vólvulo. ${ }^{5}$

Según su origen la ED se clasifica en congénita y adquirida. Cada variedad representa una entidad clínica diferente con características particulares. La ED congénita, como la presentada en este caso clínico, se produce por un defecto en el desarrollo del diafragma durante el primer trimestre del embarazo. ${ }^{6}$ Embriológicamente, la etiología de la ED se postula como la migración anormal de mioblastos de las somitas cervicales superiores en dos de las cuatro estructuras embriológicas que contribuyen al desarrollo del diafragma: el septum transverso (comenzando a las cuatro semanas de gestación) y la membrana pleuroperitoneal (a las 8 de 12 semanas de gestación). ${ }^{7,8}$

Usualmente es unilateral, es más común al lado izquierdo y puede comprometer parcial o totalmente el diafragma, en el paciente presentado se encontró compromiso total del hemidiafragma afectado. Puede presentarse como una anomalía aislada o como parte de un síndrome, p.ej., los síndromes de Kabuki, Beckwith-Wiedeman y Poland. También se han descrito casos en relación con infecciones durante el primer trimestre del embarazo como rubéola y citomegalovirus. . $^{6,9}$

Funcionalmente el diafragma afectado presenta una disminución parcial de su movilidad hasta (en los casos más severos) movimientos paradójicos durante la inspiración. En consecuencia, las manifestaciones clínicas 
varían desde cuadros aislados de taquipnea, disnea de esfuerzo o trastornos gastrointestinales inespecíficos, neumonías recurrentes o atelectasia de la base pulmonar comprimida, hasta cuadros de insuficiencia respiratoria severa. ${ }^{10}$

La ED adquirida es producida por algún trastorno en la inervación, con un músculo diafragmático estructuralmente normal. La lesión nerviosa puede tener origen central (medular) o periférica (nervio frénico) y puede ser de carácter reversible o irreversible.' Sin embargo, las causas más frecuentes son la sección accidental durante un procedimiento quirúrgico cardiovascular y el trauma obstétrico. La incidencia de parálisis frénica posterior a la realización de una cirugía cardíaca varía entre el 0.3 y el $12.8 \%$ según los diferentes reportes de casos. ${ }^{11,12}$ En ambas formas, la musculatura diafragmática está adelgazada y en su reemplazo existe tejido fibroso laxo, lo que limita su movilidad y permite su ascenso, produciendo como consecuencia limitación en la expansión pulmonar.

La ED puede presentarse de manera aislada, como en el caso que presentamos, o puede estar asociada con otros defectos congénitos, dentro de los cuales los hallazgos asociados más importantes incluyen: hipoplasia pulmonar, enfermedad cardíaca congénita, pectum excavatum, paladar hendido, hipospadias, criptorquidia y tortícolis congénita. ${ }^{13,14}$

En la serie de casos reportada por Wu et al. encontraron que la hipoplasia pulmonar $(10,5.65 \%)$ y la enfermedad cardíaca congénita $(9,5.08 \%)$ fueron las principales anomalías asociadas a pacientes con diagnóstico de eventración diafragmática $(n=177) .15$

Así como en el paciente que presentamos, las manifestaciones clínicas en pacientes con ED son inespecíficas y a menudo pueden conducir a un mal diagnóstico en un número considerable de pacientes. Algunas series de casos reportan hasta un $51.4 \%$ de pacientes asintomáticos. ${ }^{15}$

Los principales síntomas se deben a la elevación de los órganos abdominales teniendo como resultado, la compresión del lóbulo inferior del pulmón que ocasiona disnea, cianosis, dificultad respiratoria aguda, disfunción vascular y síntomas cardíacos. La anorexia, náuseas y vómitos también pueden estar asociados. La hipótesis de que la ED ocasione estos problemas podemos clasificarlos en cinco causas: a) la incapacidad para ventilar el pulmón ipsilateral, b) la compresión directa del pulmón ipsilateral, c) la neumonía debido a atelectasia crónica, d) movimiento pendular del aire entre los alvéolos (Pendelluft) debido a la ventilación entre el pulmón afectado y el pulmón no afectado, y e) el ineficiente movimiento paradójico del diafragma para realizar la ventilación del pulmón contralateral.
En el paciente que presentamos se encontró en el primer estudio radiológico un desplazamiento de las estructuras mediastinales hacia el hemitórax contralateral, esto debido a que en los lactantes el mediastino es muy móvil y el movimiento paradójico del diafragma afectado puede provocar este desplazamiento. ${ }^{16}$

En cuanto a la función pulmonar, en la ED unilateral, la capacidad vital y la capacidad pulmonar total se reducen en un 20 a 30\% como resultado de la compresión del hemitórax comprometido, de igual manera se relaciona con mayor riesgo de infecciones recurrentes del tracto respiratorio bajo y tos con o sin expectoración. En el estudio realizado por Wu et al. se encontró que en un $22.03 \%$ de los pacientes con ED presentaban infección recurrente del tracto respiratorio $(n=177){ }^{15}$

Se ha descrito también la presencia de bronquiectasias en los lóbulos pulmonares inferiores secundarios a la recurrencia de enfermedades infecciosas. ${ }^{1}$ En la ED bilateral la reducción de la función pulmonar es más importante, sobre todo en la posición supina. ${ }^{16}$

En cuanto el diagnóstico, éste se realiza con una radiografía de tórax posteroanterior y una lateral en donde se muestre una elevación del hemidiafragma derecho al menos dos espacios intercostales por arriba del izquierdo, o el izquierdo elevado un espacio intercostal por arriba del derecho. ${ }^{17}$ En casos raros de ED bilateral es difícil sospechar el diagnóstico mediante radiografía simple, ya que ambos diafragmas se encuentran elevados..$^{18}$ El estudio mediante fluoroscopia o ultrasonido son de mayor utilidad ya que permiten ver el movimiento paradójico del diafragma. ${ }^{19}$

La tomografía computada de tórax es de utilidad para corroborar los hallazgos radiográficos, identificar el grado de compresión del parénquima pulmonar subyacente, y sirve también para el diagnóstico diferencial con otras patologías de la base pulmonar o del hemiabdomen superior ipsilateral. ${ }^{10}$

El diagnóstico prenatal por ultrasonido se hace complejo y muchas veces imposible. Si la eventración es izquierda puede identificarse el estómago a la altura del corazón, el cual estará desplazado a la derecha; si ocurre en el lado derecho, el hígado se eleva hacia el tórax y puede simular una masa torácica en cortes transversales, el diagnóstico se hace demostrando la continuidad del diafragma. ${ }^{20}$

El tratamiento de la ED difiere dependiendo de su origen y manifestaciones clínicas. Es expectante en pacientes asintomáticos. Sin embargo, se sugiere que las eventraciones extensas, que ocupan gran parte del hemitórax, se deben operar por el riesgo de que interfieran con el desarrollo pulmonar posnatal. ${ }^{21}$

En los pacientes con ED congénita la indicación de corrección quirúrgica se realiza de acuerdo con la altura 
radiológica del diafragma comprometido y a la aparición de síntomas, consecuencia de la compresión del parénquima pulmonar y del desplazamiento del mediastino (figura 5).

El paciente previamente presentado en nuestro caso acudió por presentar síntomas respiratorios, en la radiografía de tórax fue posible evidenciar el hemidiafragma izquierdo superior al séptimo espacio intercostal (se encontraba a nivel de quinto espacio intercostal) por lo que el manejo quirúrgico se encontraba ya indicado pues en los pacientes asintomáticos, la indicación de cirugía se realiza cuando el diafragma comprometido se encuentra por arriba del séptimo arco costal posterior en una radiografía posteroanterior de tórax, con el fundamento de optimizar el desarrollo pulmonar y prevenir complicaciones como neumonías recurrentes o atelectasias basales, y en los pacientes sintomáticos está consensuada la corrección quirúrgica del diafragma enfermo en el momento del diagnóstico.

En los pacientes con ED adquirida, el tratamiento quirúrgico del diafragma enfermo depende del grado de insuficiencia respiratoria que provoque.

En los pacientes asintomáticos o con síntomas leves puede esperarse la recuperación del diafragma enfermo con medidas de sostén: oxigenoterapia, posición semifowler. En los pacientes sintomáticos que desarrollen insuficiencia respiratoria progresiva debe considerarse el tipo de lesión nerviosa, misma que podemos clasificar como lesión reversible e irreversible.

En los pacientes con lesión reversible se deberá evaluar evolución clínica de la disfunción respiratoria y radiológica, posteriormente la mejoría clínica se certifica con la corrección de los gases en sangre y el descenso radiológico del diafragma. Ante una evolución desfavo- rable, se determina el ingreso del paciente a asistencia respiratoria mecánica (ARM) con presión positiva continua en la vía aérea (CPAP); este método produce un descenso y estabilización del diafragma y aumento del volumen pulmonar. Si se toma esta conducta, evaluar la posibilidad de destete del respirador en un plazo no mayor a 7 a 10 días y en caso de que esto mismo no ocurra, es decir no reaparece actividad diafragmática luego de 7 a 10 días de producida la lesión, se sugiere la corrección quirúrgica. Por último no se recomienda la permanencia prolongada en ARM debido a las complicaciones propias del método.

Ahora bien, en los pacientes con patología cardíaca residual o enfermedad respiratoria concomitante, es conveniente determinar en qué grado contribuye cada afección a la insuficiencia respiratoria del paciente para poder determinar el momento oportuno para el descenso quirúrgico del diafragma. Si un paciente logra una franca mejoría de la disfunción respiratoria mediante el descenso diafragmático con CPAP, es esperable un resultado igualmente favorable con la corrección quirúrgica, aun en presencia de enfermedad concomitante. Por el contrario, si con el descenso diafragmático logrado con CPAP no se alcanza una mejoría significativa de la insuficiencia respiratoria es evidente que coexisten factores cardíacos y/o respiratorios. En este caso, el tratamiento quirúrgico del diafragma se realizará en forma coincidente o diferida con el tratamiento de la enfermedad cardiorrespiratoria acompañante.

En aquellos pacientes con lesión irreversible, si la sección accidental del nervio frénico es advertida en el acto quirúrgico al momento de producida, se recomienda la reparación directa del nervio seccionado mediante

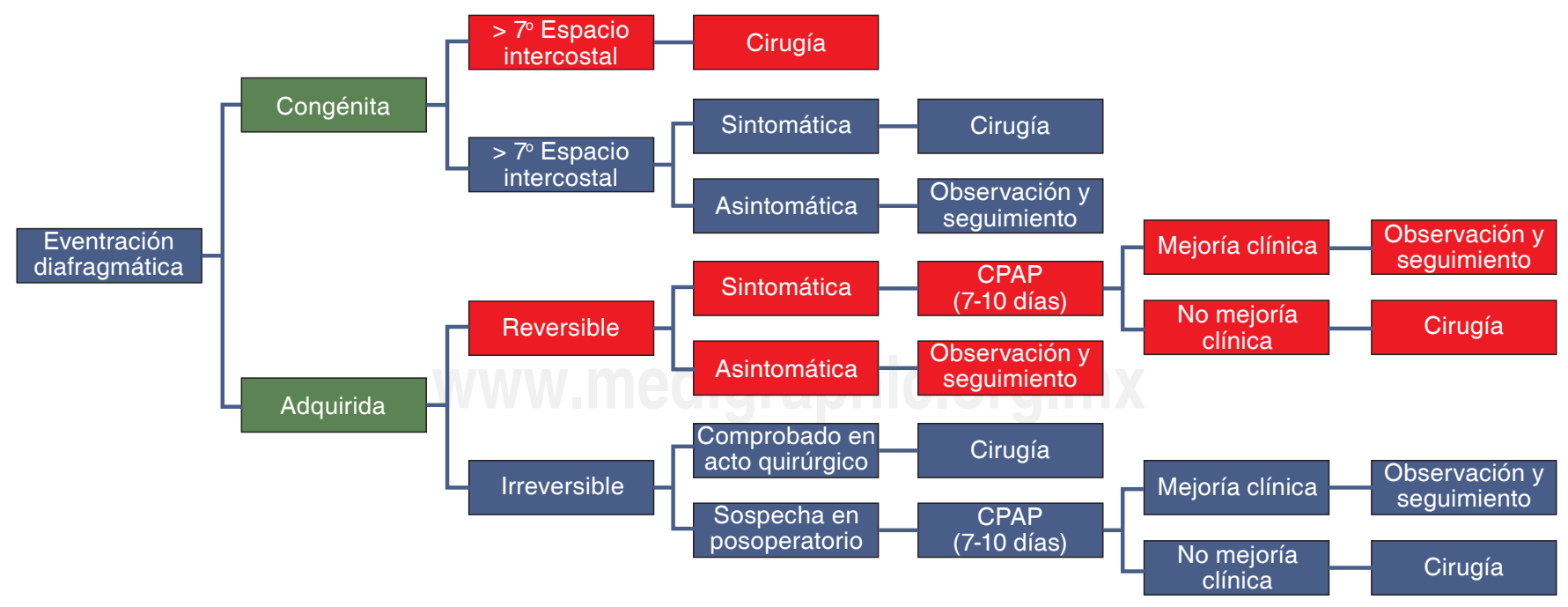

Figura 5. Indicación quirúrgica de eventración diafragmática. Modificado de Garrido P, Acastello E. Eventración diafragmática en la edad pediátrica. Rev Med Clin Condes 2009;20(6):776-781. 
la aproximación de sus extremos; siempre seguida de una plastía diafragmática debido a que la reaparición de la movilidad diafragmática luego de la neurorrafia puede demorar varios meses. Si en un posoperatorio de cirugía torácica o cardíaca se detecta diafragma elevado y se sospecha lesión del nervio frénico se debe evaluar si existe mejoría de la disfunción respiratoria con CPAP y/o la posibilidad de destete de la ventilación mecánica, si esto no se produce debe realizarse el descenso quirúrgico del diafragma en forma temprana.

Si existe patología cardíaca o enfermedad respiratoria concomitante es prioritario evaluar la afección dominante y definir las prioridades del tratamiento antes de decidir la corrección quirúrgica del diafragma. ${ }^{10}$

\section{CONCLUSIONES}

La ED es una patología poco frecuente en la población pediátrica, en la mayor parte de los casos cursa asintomática o con síntomas respiratorios inespecíficos y su diagnóstico puede ser incidental. El caso presentado corresponde a una ED congénita, misma que se produce por un defecto intrínseco del músculo diafragmático a diferencia de la ED adquirida la cual se origina por trastornos en la inervación siendo las causas más frecuente las lesiones del nervio frénico. Así como en nuestro paciente, en la mayor parte de los casos el tratamiento de la ED es quirúrgico, por lo que es de gran importancia saber determinar el momento oportuno para la cirugía, favoreciendo de esta manera la adecuada evolución y minimización de complicaciones que esta patología pueda ocasionar.

\section{REFERENCIAS}

1. Majluf R. Enfermedades del diafragma. En: Macri C, Teper A, eds. Enfermedades respiratorias pediátricas. Buenos Aires: McGraw Hill-Interamericana; 2003. p. 751-753.

2. Willcox PG, Pardy RL. Diaphragmatic weackness and paralysis. Lung 1989;167:323-341.

3. Thomas TV. Congenital eventration of the diaphragm. Ann Thorac Surg 1970;10(2):180-192.

4. Jurcak-Zaleski S, Comstock CH, Kirk JS. Eventration of the diaphragm. Prenatal diagnosis. J Ultrasound Med 1990;9(6):351-354.

5. Contreras El, Escobar HR, Necochea KMC, Castro MS, Sánchez DI. Tres casos de parálisis diafragmática: Utilidad del estudio electromiográfico. Rev Chil Pediatr 2004;75(1):48-54. Disponible en: http://dx.doi. org/10.4067/S0370-41062004000100007

6. Deslauriers J. Eventration of the diaphragm. Chest Surg Clin N Am 1998;8(2):315-530.

7. Schumpelick V, Steinau G, Schlüper I, Prescher A. Surgical embryology and anatomy of the diaphragm with surgical applications. Surg Clin North Am 2000;80(1):213-239, xi.

8. Thomas TV. Nonparalytic eventration of the diaphragm. J Thorac Cardiovasc Surg 1968;55(4):586-593.

9. Kulkarni ML, Sneharoopa B, Vani HN, Nawaz S, Kannan B, Kulkarni PM. Eventration of the diaphragm and associations. Indian J Pediatr 2007;74(2):202-205.

10. Garrido P, Acastello E. Eventración diafragmática en la edad pediátrica. Rev Med Clin Condes 2009;20(6):776-781.

11. Akay TH, Ozkan S, Gultekin B, et al. Diaphragmatic paralysis after cardiac surgery in children: incidence, prognosis and surgical management. Pediatr Surg Int 2006;22(4):341-346.

12. Joho-Arreola AL, Bauersfeld U, Stauffer UG, Baenziger $\mathrm{O}$, Bernet V. Incidente and treatment of diaphagmatic alter cardiac surgery in children. Eur J Cardiothorac Surg 2005;27(1):53-57.

13. Smith CD, Sade RM, Crawford FA, Othersen HB. Diaphragmatic paralysis and eventration in infants. J Thorac Cardiovasc Surg 1986;91(4):490-497.

14. Kapoor V, Wright IM. Congenital myotonic dystrophy with cardiac conduction defect and eventration of the diaphragm. Pediatr Int 2010;52(1):e6-e8. doi: 10.1111/j.1442-200X.2009.02998.x.

15. Wu S, Zang N, Zhu J, Pan Z, Wu C. Congenital diaphragmatic eventration in children: 12 years' experience with 177 cases in a single institution. J Pediatr Surg 2015;50(7):1088-1092. doi: 10.1016/j.jpedsurg.2014.09.055.

16. Sodhi KS, Narsimhan KL, Bhattacharya A, Khandelwal N. Bilateral congenital diaphragmatic eventration: an unusual cause of respiratory distress in an infant. Afr J Paediatr Surg 2011;8(2):259-260. doi: 10.4103/01896725.86082

17. Gómez-Ruiz A, Medina-Vega FA, Tejeda-Tapia HD. Eventración diafragmática bilateral: informe de un caso y revisión de la literatura. Acta Pediatr Mex 2013;34(3):161-165.

18. Zoffer S, Stolar C. Eventration of the diaphragm. In: Mattei P, editor. Surgical directives: pediatric surgery. Philadelphia: Lippincott, Williams and Wilkins; 2003. p. 527-528.

19. Tracy T, Luks F. Diaphragmatic hernias and eventration. In: Ziegler, editor. Operative pediatric surgery. Philadelphia: McGraw Hill; 2003. p. 495-499.

20. Velázquez PS, Araluce BJ, Velázquez RG, Araluce CM, Trinchet SRM, Figueredo BRJ. Eventración diafragmática en el recién nacido. CCM 2015;19(3):548-555.

21. Sarihan H, Cay A, Akyazici R, Abes M, Imamoglu M. Congenital diaphragmatic eventration: treatment and postoperative evaluation. J Cardiovasc Surg (Torino) 1996;37(2):173-176.

\section{$\triangle$ Correspondencia:}

Dr. Carlos Guzmán Valderrábano,

Hospital Infantil de Especialidades del estado

de Chihuahua, México.

Correo electrónico: dr_valderrabano@hotmail.com drcarlosv@pediahero.com.mx

Los autores declaran no tener conflicto de intereses. 